\title{
Single Parameter Model Of Minimal Surface Construction For Dynamic Garment Pattern Design *
}

\author{
Roger $\mathrm{Ng}^{1+}$, Winnie Yu ${ }^{1}$ and L.F. Cheung ${ }^{1}$ \\ ${ }^{1}$ Institute of Textiles and Clothing, Hong Kong Polytechnic University \\ ${ }^{2}$ Department of Mathematics, Chinese University of Hong Kong
}

( Received 4 October 2006, accepted 4 February 2007)

\begin{abstract}
Dynamic ease allowance is the amount of spacing between the garment and the wearer that is required to allow the wearer to performance certain posture. Without sufficient dynamic ease allowance, the motion of the wearer will be restricted. In this article, the authors present a single parameter model, which aims at calculating the minimal surface needed for performing some required postures. In this model, the boundary conditions are imposed at the wrist and waist. An additional collocation condition is imposed at the armhole. The minimal surface must pass through both end points (wrist and waist) and the collocation point (armhole). The formulation will be presented and compared with physical data.
\end{abstract}

Keywords: garment pattern design, minimal surface, dynamic ease.

\section{Introduction}

In garment pattern design, the extra spacing between the garment and the wearer is called the ease allowance. There are three different types of ease allowances according to different functions. Firstly, the basic movements, such as breathing and sitting, require static ease allowance. Secondly, extreme postures, such as raising a toe to hit one's head, needs dynamic ease allowance. Thirdly, the garment itself needs the extra spacing to conform the required silhouette. These eases are also referred as comfort ease, movement ease and styling ease respectively.

In this article, the focus is on the modeling of the dynamic ease allowance and to find out the shape of the garment pattern that allows the wearer to perform certain extreme postures. From the theoretical point of view, the garment is considered as a surface. The shape of the surface can impose restrictions on the motion of the wearer. An optimal shape of the surface can be calculated to maximize the motions of the wearer. The project proceeded with the trial selection of extreme postures with the assistance of a yoga expert. After the selection, 20 subjects were invited to perform the posture while their movements were recorded by a motion capturing system. The data was analyzed and compared with the theoretical prediction as part of the verification process.

\section{Literature Review}

In the literature, determination of dynamic ease allowance is often disguised as the fitting problem or kinetic comfort. If a wearer considers the garment fit to be comfortable, it must have the appropriate amount of dynamic ease allowance. Based on qualitative techniques and/or statistical analysis, many researchers attempted to study the property of dynamic ease allowance. Prevatt [1] asked the subjects to wear selected protective garments to perform a series of postures and body movements. Their comments were collected for analysis. Larmour [2] studied the best-fit garment for senior citizens between the ages of 65 and 74, based on the body measurements. Similarly, Burke [3] developed four different prototypes of fit-modified garment for ambulatory women between 68 and 94 years of age to identify the best-fit prototypes. Cho [4] designed and studied the mobility of the hospital gowns, through the interviews and survey of the female nurses. Furthermore, range-of-motion and fit are evaluated subjectively and statistically, such as [5], [6], [7],

\footnotetext{
* The work described in this paper was fully supported by a grant from the Research Grants Council of the Hong Kong Special Administrative Region, China (Project No. 5284/03E).

+ Roger Ng. Tel.: +852-2766 6532; fax: +852-2773 1432.

E-mail address: roger.ng@polyu.edu.hk.
} 
[8], [9], [10]. Fit can also be measured graphically by moire topography [11]. All of these studies are experimental in nature. The theoretical formulation of the ease allowance with respect to the postures has not been reported.

\section{Posture Definition}

In biomechanics, one can reach only limited space and the trajectories of such maximum reachable points form the range-of-motion. Such range-of-motion forms an envelop surface. The complete range-ofmotion is the union of range-of-motion of each part of the body. Two examples of selected postures are shown in Fig. 1.

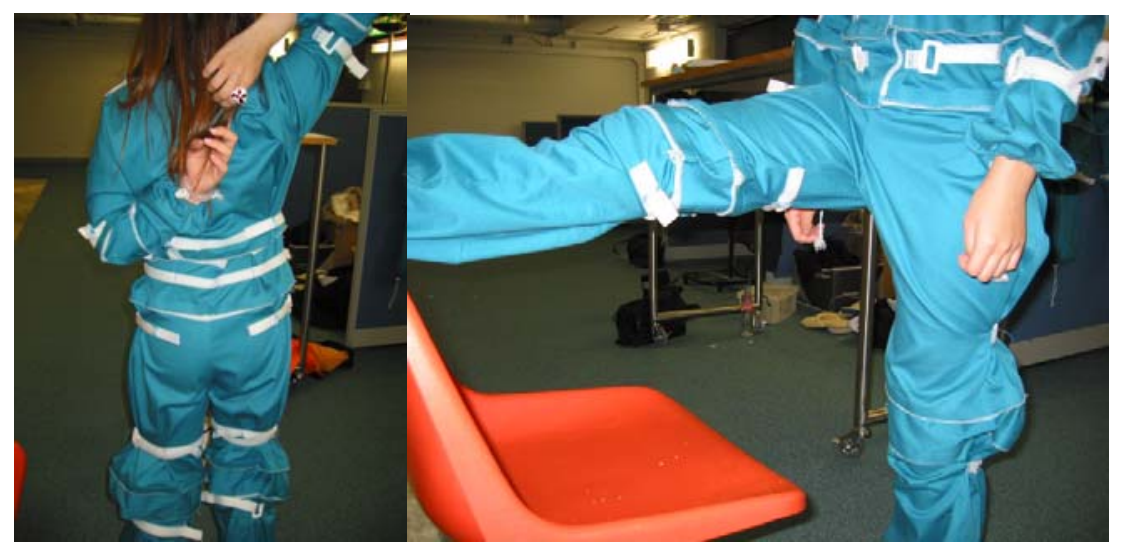

Fig. 1. Sample Postures

The extreme postures may vary slightly with different subjects, because each subject has different level of stretching. For ensuring the safety of the experiment, a yoga master was invited to demonstrate the extreme postures. The yoga master reported the level of stretching and which muscles are under stretch. The comments were compiled into a precaution procedure.

These extreme postures can be classified into 2-D postures and 3-D postures. In 2-D postures, the movements are restricted on a motion plane, while 3-D postures are not restricted at all. In this article, the focus is on the 2-D posture, as one parameter is typically sufficient to describe this class of postures. It is mainly because many of the motions can be partitioned according to the joints of the human subject. Once the origin of the local coordinate system is defined at the joint, the motions of the limbs are reduced to a local plane containing the limbs. These basic motions are thus the simple cases and they can be combined as the building blocks of the more complicated cases.

\section{Single Parameter Model}

The hand is composed of the upper and lower arm. They are covered by a sleeve. The sleeves can be set-in or grown-on. Set-in sleeve means that the sleeve is a separate piece of fabric sewn to the bodice of the garment at the armhole while the grown-on sleeve is an extension of the bodice. Therefore, the generic sleeve is defined to cover the arm from shoulder to wrist. The shape of the sleeve can restrict the movement of the arm. The main cause of the restriction is because of the shape of the armhole of the sleeve. Fig. 1 and 2 demonstrate the cause and effect of the armhole shape. The size of the armhole is bigger in Fig. 1 and the sleeve restricts the movement, while the size of the armhole is smaller in Fig. 2 and the sleeve allows the raising up of the hand. This problem can be formulated mathematically as shown in Fig. 3. The shape of the sleeve can be restricted at the armhole, which is the key parameter that is to be derived. The variables are defined in Table I. 


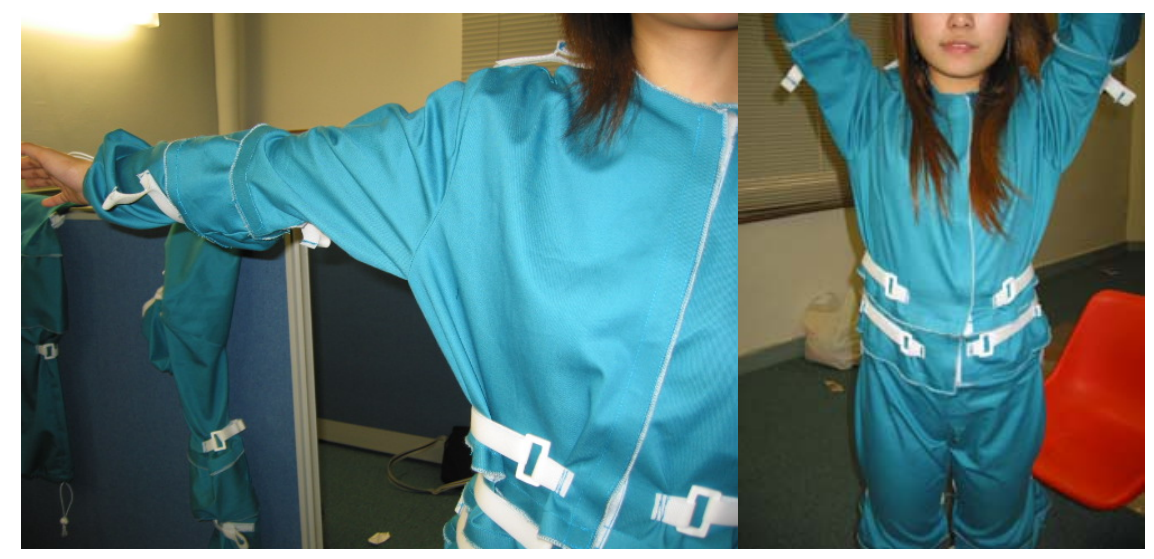

Fig. 2. Restricted Movement

Fig. 3 Free Movement

Table 1 IDEFINITIONS OF VARIABLES

\begin{tabular}{|c|c|c|c|}
\hline \multicolumn{2}{|c|}{ Variable ID } & Type & Meaning \\
\hline$a h u$ & $\left(x_{a h u}, y_{a h u}\right)$ & Point & Under arm point of the armhole (garment) \\
\hline ARC & & Function & Arc length of a curve \\
\hline AREA & & Function & Area bounded by a polygon or Area under a curve and the $x$-axis \\
\hline $\mathrm{B}_{\mathrm{ARM}}$ & & Function & Area minimizing Bezier curve of the under side of the sleeve \\
\hline cuff & & Scalar & Width of wrist (garment) \\
\hline dea & & Scalar & Dynamic ease allowance measured by linear measurement \\
\hline $\operatorname{dea} 2 \mathrm{D}$ & & Scalar & Dynamic ease allowance measured by the cross-sectional area \\
\hline scye & & Scalar & Length of the armhole and $\|$ sht - shu $\|<=$ scye \\
\hline$s l$ & & Scalar & Sleeve length of the top side from sht to wrt (garment) \\
\hline slu1 & & Scalar & Sleeve length of the under side from $a h u$ to $w a$. (garment) \\
\hline slu2 & & Scalar & Sleeve length of the side seam from $a h u$ to wru. (garment) \\
\hline sht & $\left(x_{\text {sht }}, y_{\text {sht }}\right)$ & Point & Top shoulder point (body) \\
\hline shu & $\left(x_{\text {shu }}, y_{\text {shu }}\right)$ & Point & Under shoulder point (body) \\
\hline SU1 & & Curve & Under side sleeve curve from $w a$ to $a h u$ (garment) \\
\hline SU2 & & Curve & Under side sleeve curve from ahu to wru (garment) \\
\hline$w a$ & $\left(x_{w a}, y_{w a}\right)$ & Point & Side seam point of the waist (garment and body) \\
\hline wrt & $\left(x_{w r t}, y_{w r t}\right)$ & Point & Top arm point of the wrist girth (garment and body) \\
\hline wru & $\left(x_{w r u}, y_{w r u}\right)$ & Point & Under arm point of the wrist girth (garment) \\
\hline$\theta$ & & Angle & Reach Angle, angle between the line wrt - sht and $x$-axis \\
\hline
\end{tabular}

\section{Formal Definition of the Problem}

In this section, the raising arm problem is modeled by an in-plane motion along the frontal plane with two rods (upper and lower arms). The origin of the local coordinate system is defined at the shoulder point, which also serves as the joint of the rod (upper arm) and the body. The $x$-axis is the horizontal direction while the $y$-axis is the vertical direction.

$$
\text { sht }=\left(x_{\text {sht }}, y_{\text {sht }}\right)=(0,0)
$$

The arm has a thickness of (sht - shu) which is less than or equal to the scye, or else either the fabric is stretchable or the sleeve will run into the arm.

$$
\| \text { sht }- \text { shu } \|<=\text { scye }
$$

The position of the $a h u$, which is the controlling parameter to be determined, falls within the circle or radius scye from sht.

$$
\| \text { sht }-a h u \|=\text { scye }
$$


The $a h u$ is connected to the side waist line, which is assumed to be a fixed point $w a$, by the side seam of the garment. The shape of the side seam is defined by the function SU1 $(t)$ over the domain of $[0,1]$. So, the arc length of SU1(t) is equal to distance between wa and $a h u$.

$$
\|w a-a h u\|=\operatorname{ARC}(\operatorname{SU} 1(t))
$$

The $a h u$ is also connected to the under side of the cuff or wrist, which is assumed to be a fixed point wru, by the under side of the seam. The shape of the side seam is defined by the function SU2 $(t)$ over the domain of $[0,1]$. So, the arc length of SU2 $(t)$ is equal to distance between $a h u$ and $w r u$.

$$
\|a h u-w r u\|=\operatorname{ARC}(\operatorname{SU} 2(t))
$$

When the arm is raised, the top side of the cuff or wrist, wrt, is rotated about the shoulder point, sht. In turn, the underside of the cuff, wru, is rotated by the same angle.

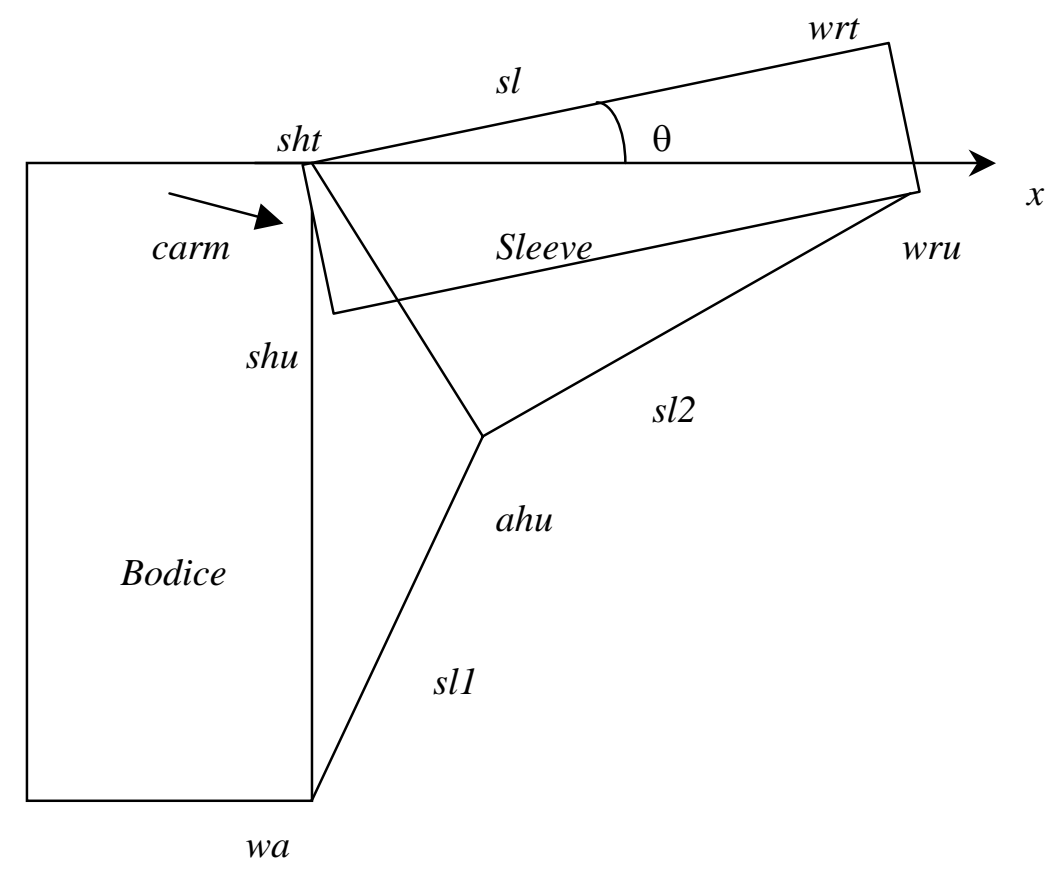

Fig. 3 Rods model of raising arm problem

Assuming the cuff will always make a right angle to the arm, (6) can be written down.

$$
(w r t-s h t) .(w r t-w r u)=0
$$

Based on (1) to (6), the relationship between the reach angle $\theta$, formed by the vector (sht - wrt) and the $x$-axis can be expressed explicitly when SU1(t) and SU2(t) are straight lines, and can be numerically determined when SU1(t) and SU(2) are higher order curves.

\subsection{First order solution}

When the arm is being raised, the pivot point is the shoulder point, sht, and the hanging point is the wrt. The wrt supports the wru at the right angle. Then, wru takes up the role of a hanging point. When the sleeve length is given, the position of $a h u$ can be determined by the intersection of two circles, one centered at sht with a radius of scye and another one centered at $w a$ with a radius of slu, and selecting the solution closer to sht. Since the position of wru can be expressed as a function of the reach angle $\theta$, the required relationship is established. The command for generating the explicit form in Mathematica ${ }^{\mathrm{TM}}$ is given in Table II. The dynamic ease function can then be defined by the associated parameters of $s l, \theta$, wa, cuff, su1, su2. 
Table 2. DERIVING DYNAMIC EASE FUNCTION FOR ARM RAISING PROBLEM

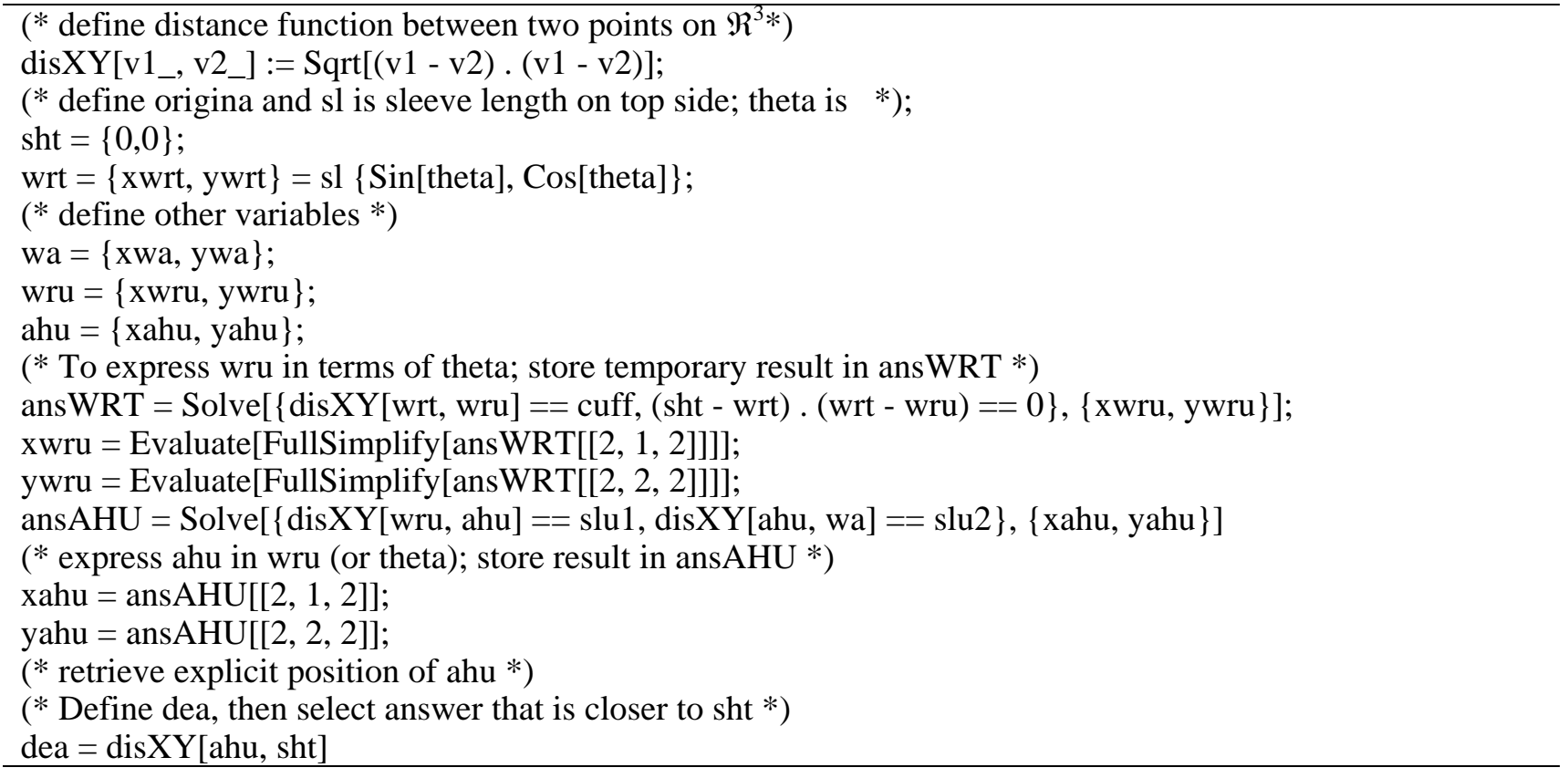

\subsection{Higher order solution}

The relationship between the dynamic ease (or position of the $a h u$ ) and the reach angle has been derived in section A. This analysis is valid whenever either the under side sleeve curve is a straight line, or the under side sleeve curve has been fully stretched into a straight line. However, the under side sleeve curve does not have to be a straight line, because if the area bounded between the body and the sleeve can be minimized, the uniform is less bulky. The trial function for this area minimization problem is chosen to be the family of cubic Bezier curves, that is defined by (7) and satisfying the requirements stated in (8) - (13). The control vertices are labeled as $p_{0}$ to $p_{3}$. The curve parameter is $t$. The end point must match at the waist point $w a$, and the under side of the cuff, wru. Based on the hull property of Bezier curve, the control vertex $p_{1}$ is forced to be located along the direction from wa to carm, which is the center of the arm, while the other control vertex $p_{2}$ is forced to be located along the direction from wru to carm. This curve must also pass through the $a h u$ point at some value of $t_{a h u}$ to achieve the movement requirement. Finally, dynamic ease allowance, dea2D, can be defined as the area bounded by the region wa-carm-wru and $\mathbf{B}_{\text {arm }}$ (13). Since the local coordinate system is set at the sht, depending on the angle $\theta$, the under side of the sleeve may intercept the $x$-axis and the area must be split into two regions.

$$
\begin{gathered}
\mathbf{B}_{\text {arm }}\left(p_{0}, p_{1}, p_{2}, p_{3} ; t\right)=p_{0} t^{3}+p_{1} t^{2}(1-t)+p_{2} t(1-t)^{2}+p_{3}(1 \mathrm{t})^{3} \\
p_{0}=w a \\
p_{1}=w a+s_{1}(w a-c a r m) \\
p_{2}=w r u-s_{2}(w r u-c a r m) \\
p_{3}=w r u \\
\mathbf{B}_{\text {arm }}\left(p_{0}, p_{1}, p_{2}, p_{3} ; t_{a h u}\right)=a h u \\
\operatorname{dea} 2 D=\operatorname{AREA}(w a, c a r m, w r u)-\mid \operatorname{AREA}\left(\mathbf{B}_{\text {arm }}\left(\mathrm{s}_{1}, s_{2} ; t=0 \text { to } 1\right)\right) \mid
\end{gathered}
$$

Since AREA(wa, carm, wru) is constant, the minimization problem can be reduced to the minimization of $\operatorname{AREA}\left(\mathbf{B}_{\mathbf{a r m}}\left(\mathrm{s}_{1}, s_{2} ; t=0\right.\right.$ to 1$\left.)\right)$. There are two parameters that can be varied, namely $\mathrm{s}_{1}$ and $s_{2}$.

Before applying the constraint (12), the explicit form of the kernel is shown in (14).

$$
\begin{aligned}
\operatorname{KERNEL}\left(s_{1}, s_{2}, t\right)= & 3\left(t\left(s_{1}(-2+3 t)\left(\mathrm{x}_{\text {carm }}-\mathrm{x}_{\mathrm{wa}}\right)-2(-1+\mathrm{t})\left(\mathrm{x}_{\mathrm{wa}}-\mathrm{x}_{\mathrm{wru}}\right)\right)\right. \\
& \left.+\mathrm{s}_{2}\left(1-4 \mathrm{t}+3 \mathrm{t}^{2}\right)\left(\mathrm{x}_{\text {carm }}-\mathrm{x}_{\mathrm{wru}}\right)\right)\left(\mathrm{t}^{3} \mathrm{y}_{\mathrm{wa}}-3(-1+\mathrm{t}) \mathrm{t}^{2}\left(\mathrm{y}_{\mathrm{wa}}+\mathrm{s} 1\left(-\mathrm{y}_{\text {carm }}+\mathrm{y}_{\mathrm{wa}}\right)\right)\right. \\
& \left.-(-1+t)^{3} y_{\text {wru }}+3(-1+t)^{2} t\left(\mathrm{~s}_{2}\left(y_{\text {carm }}-y_{\text {wru }}\right)+\mathrm{y}_{\mathrm{wru}}\right)\right)
\end{aligned}
$$

Before applying the constraint (12), the area function is shown in (15).

$$
\begin{aligned}
\operatorname{AREA}\left(w a, c a r m, \text { wru, } \mathrm{s}_{1}, \mathrm{~s}_{2}\right) & =1 / 20\left(3 \mathrm { s } _ { 1 } ( - 2 + \mathrm { s } _ { 2 } ) \left(-\mathrm{x}_{\mathrm{wru}} \mathrm{y}_{\text {carm }}-\mathrm{x}_{\text {carm }} \mathrm{y}_{\mathrm{wa}}+\mathrm{x}_{\mathrm{wru}} \mathrm{y}_{\mathrm{wa}}+\mathrm{x}_{\mathrm{wa}}\left(\mathrm{y}_{\mathrm{carm}}-\mathrm{y}_{\mathrm{wru}}\right)\right.\right. \\
& \left.+\mathrm{x}_{\text {carm }} \mathrm{y}_{\mathrm{wru}}\right)-2\left(-5\left(\mathrm{x}_{\mathrm{wa}}-\mathrm{x}_{\mathrm{wru}}\right)\left(\mathrm{y}_{\mathrm{wa}}+\mathrm{y}_{\mathrm{wru}}\right)\right.
\end{aligned}
$$




$$
\left.\left.+3 \mathrm{~s}_{2}\left(\mathrm{x}_{\mathrm{wru}} \mathrm{y}_{\text {carm }}+\mathrm{x}_{\text {carm }} \mathrm{y}_{\mathrm{wa}}-\mathrm{x}_{\mathrm{wru}} \mathrm{y}_{\mathrm{wa}}-\mathrm{x}_{\text {carm }} \mathrm{y}_{\mathrm{wru}}+\mathrm{x}_{\mathrm{wa}}\left(-\mathrm{y}_{\mathrm{carm}}+\mathrm{y}_{\mathrm{wru}}\right)\right)\right)\right)
$$

After applying for the constraint (12), the relationship between $s 1$ and $s 2$ can be found. The area function then depends only on one parameter. However, the explicit form is very complicated and can be solved numerically.

At this stage the 2-D minimal surface, the area minimizing curve has been found. Together with the upper sleeve curve, the 3-D sleeve surface can be mounted on the arm.

Table 3. SOLVING THE AREA MINIMIZING CURVE OF THE UNDER SIDE OF THE SLEEVE

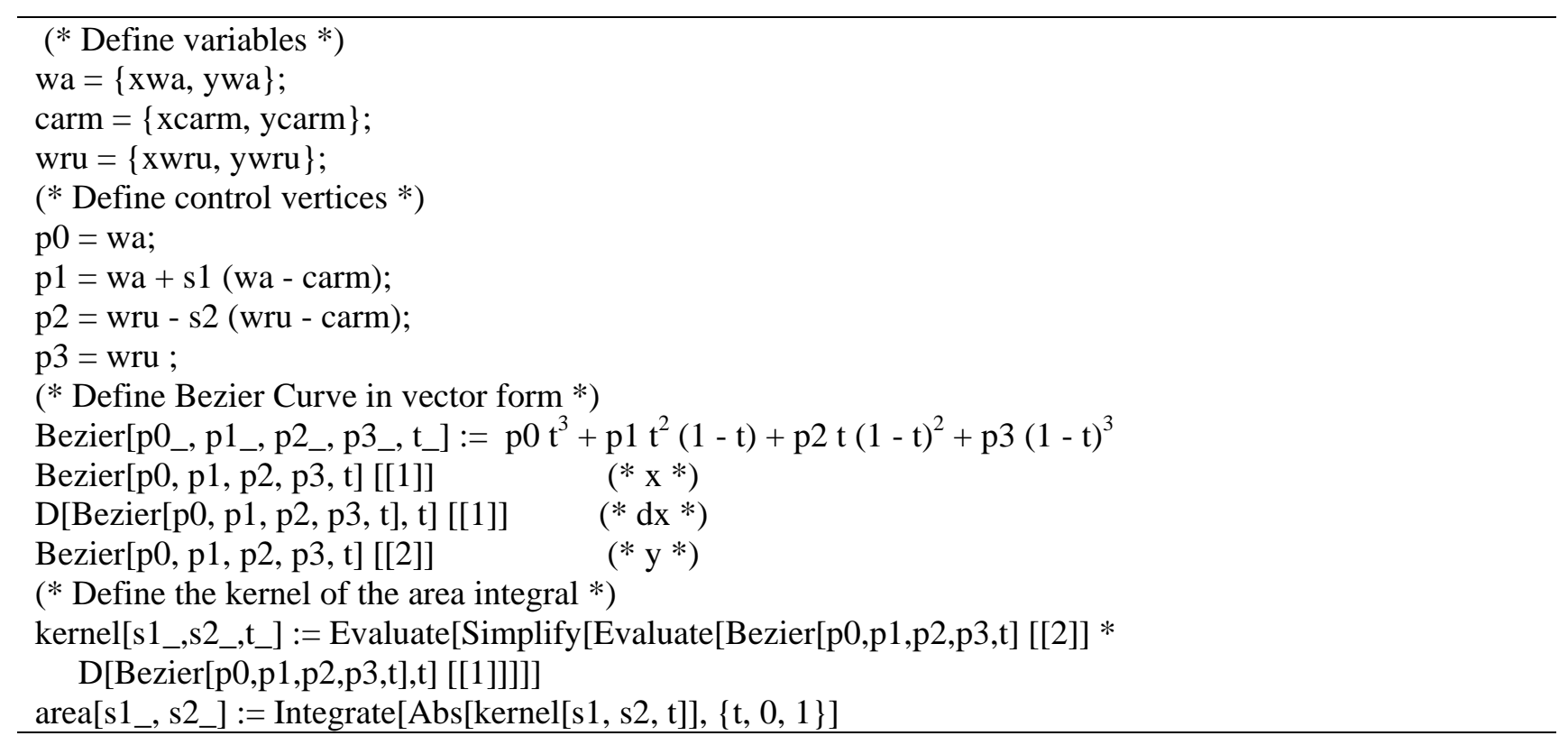

\section{Data collection and verification}

Twenty subjects of age ranging from 19 to 21 years ago were invited to participate in the research. Their body measurements were recorded manually. A set of modularly designed cover all garments can be set up to match the individual body measurements. Each subject needed to raise their arm as high as possible. The movements were recorded by a motion capturing system and compared to the first order solution. Some errors have been observed. However, if the measurements were taken as a straight line, rather than along the sleeve surface, the accuracy is higher. The source of error is most likely the fact that the sleeve cannot be fully stretched into a straight line (Fig. 2). In principle, the data collected conform to the prediction.

\section{Conclusion}

A piece of garment can restrict the movement of the wearer. The relationship between the reach angle and the sleeve length has been derived. The position of the point ahu (under point of the armhole) is the crucial parameter that determines the dynamic ease allowance. Experiments have been conducted for the verification of the derived model. The result is reasonable, and the source of error most likely comes from the fact that the sleeve of the trial cover all garment cannot be stretched fully into a straight line. Therefore, the experiment does not fully satisfy the condition of the experiment. Nevertheless, the first order solution is a reasonable approximation.

Furthermore, the model of the refined shape of the sleeve that can minimize the ease allowance (crosssectional area bounded by the body and the garment) has been derived. The trial function is a cubic Bezier curve satisfying constraints of (8) - (12). The area function can be expressed in terms of single parameter, $s_{1}$ or $s_{2}$. It can be solved numerically. The 2-D minimal surface, which is the area minimizing curve $\mathbf{B}_{\text {arm }}$, has been found. Together with the curve of the top side of the sleeve and the surface of the arm, one can mount a 3-D sleeve surface.

Future work of this project includes: (1) the extension of 2-D analysis to 3-D, (2) the extension of the model to the lower part of the body, namely the leg spreading problem and (3) development of construction principle of pattern design based on the dynamic ease allowance. 


\section{References}

[1] M. B. Prevatt. Fit and sizing evaluation of limited-use protective coveralls (garment fit), PhD Thesis, Virginia Polytechnic Institute and State University, 1991.

[2] M. S. Larmour. A study of body measurements relating to the fit of clothing for 65 to 74 year old women, MSc thesis, University of Arizona, 1988.

[3] B. F. Burke. Satisfaction of women over 65 years of age with a fit-modified garment (women elderly), PhD thesis, Texas Woman’s University, 1994.

[4] K. Cho. User-centered design and evaluation of functional hospital gowns, PhD thesis, Kansas State University, 2001.

[5] P. S. Adams, W.M. Keyserling. The effect of size and fabric weight of protective coveralls on range of gross body motions. American Industrial Hygiene Association Journal. 1995, 56(4): 333-40.

[6] S. P. Ashdown, M. DeLong. Perception testing of apparel ease variation. Applied Ergonomics. 1995, 26(1): $47-54$.

[7] P. Chan, H. Tam, A. Mak, K.K. Chan. A test procedure for measurement of movement allowance for preschool children clothing. Proc. of the $3^{\text {rd }}$ Asian Textile Conference. 1995, 343-347.

[8] J. E. Smith. The evaluation and optimization of sensorial comfort, PhD thesis, University of Salford, 1987.

[9] H. T. Chen. Factors affecting perception of fit of jeans, MSc thesis, University of North Texas, 1987.

[10] J. Huck. Protective clothing systems: a technique for evaluation restriction of wearer mobility. Applied ergonomics. 1988, 19(3): 185-190.

[11]W. Yu. Fit Evaluation of Men’s Jacket using Moiré Topography. Textile \& Clothing. 2000, 12(2).

\section{Trademark}

Mathematica $^{\mathrm{TM}}$ is a trademark of Wolfram Research Inc. 
